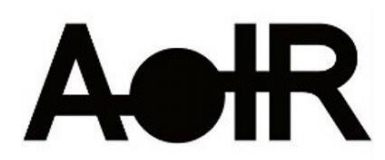

Selected Papers of \#AolR2021:

The 22nd Annual Conference of the

Association of Internet Researchers

Virtual Event / 13-16 Oct 2021

\title{
A Critical Future of Virtual Reality: All Work and No Play
}

\author{
Marcus Carter \\ Socio-Tech Futures Lab, The University of Sydney \\ Ben Egliston \\ Digital Media Research Centre, Queensland University of Technology
}

\section{The Platformization of Virtual Reality}

Head-Mounted Virtual Reality (VR) is generally discussed as a gaming technology, but in this paper we argue for the greater need to take VR seriously as a general computing platform and future site for, and of, work. For Facebook (who hold a dominant $39 \%$ share of the VR market) VR is not simply a gaming device but - as we unpack elsewhere - "a new frontier for social media", framed by Zuckerberg as a "new kind of social computing platform" (Egliston \& Carter, 2020). Oculus now operates within Facebook under the umbrella of its 'Facebook Reality Labs' (FRL) technological development wing, which pursues research projects in both VR and AR computing with overarching goals of seeing these emerging technologies adopted ubiquitously. An example of this is Facebook's 'Infinite Office' technology (see Figure 1) that imagines a flexible, mobile virtual office space for traditional desktop work as well as meetings via VR telepresence.

\section{Approach and Methods}

Here, we report on our research that is examining the implications of VR as (1) a technology being introduced into workplaces; as (2) a possible site for existing forms of labour, in technologies like Infinite Office; and as (3) a platform for transformed and new kinds of labour, such as remote work via telepresence. We study these sites drawing on methods established in political economy of communication research that utilize 'trade publications' to examine media industries (see Corrigan, 2018; Wasko, 2004; Carter \& Egliston, 2021), which have recently been fruitfully applied in internet studies (Wilken, Burgess, \& Albury, 2019). In doing so, we highlight the immediate surveillant and 
discriminatory issues, and identify the ways that VR may be utilized to undermine worker rights and working conditions.

\section{Algorithmic Discrimination in VR Enterprise Training}

One emerging site for VR in the workplace is in workplace training and assessment, a deployment vulnerable to algorithmic discrimination. For instance, in 2019 Walmart announced its partnership with VR start-up STRIVR, describing how they have been using interactive VR scenarios to train employees and for the hiring process. In related news coverage, they describe the promotion - and $10 \%$ pay rise - for a 12-year employee based on performance in the VR training. While Walmart emphasise that VR assessment is only one of the "data points" used in hiring decisions, the VR companies we examined enthusiastically frame the potential for the complete automation of hiring decisions based on the "objective" data collected from VR use.

Claims like these echo existing concerns with the use of learning analytics to categorize and assess, and algorithmic decision making more generally (Andrejevic, 2019). VR data is of course not "objective"; while we found no discussion of the training datasets in our study (Carter \& Egliston, 2021), commercial machine-learning products are typically trained on biased datasets of neurotypical, male, and able-bodied engineers (Mehrabi et al. 2019). In the context of VR learning analytics, this has the potential to be a form of

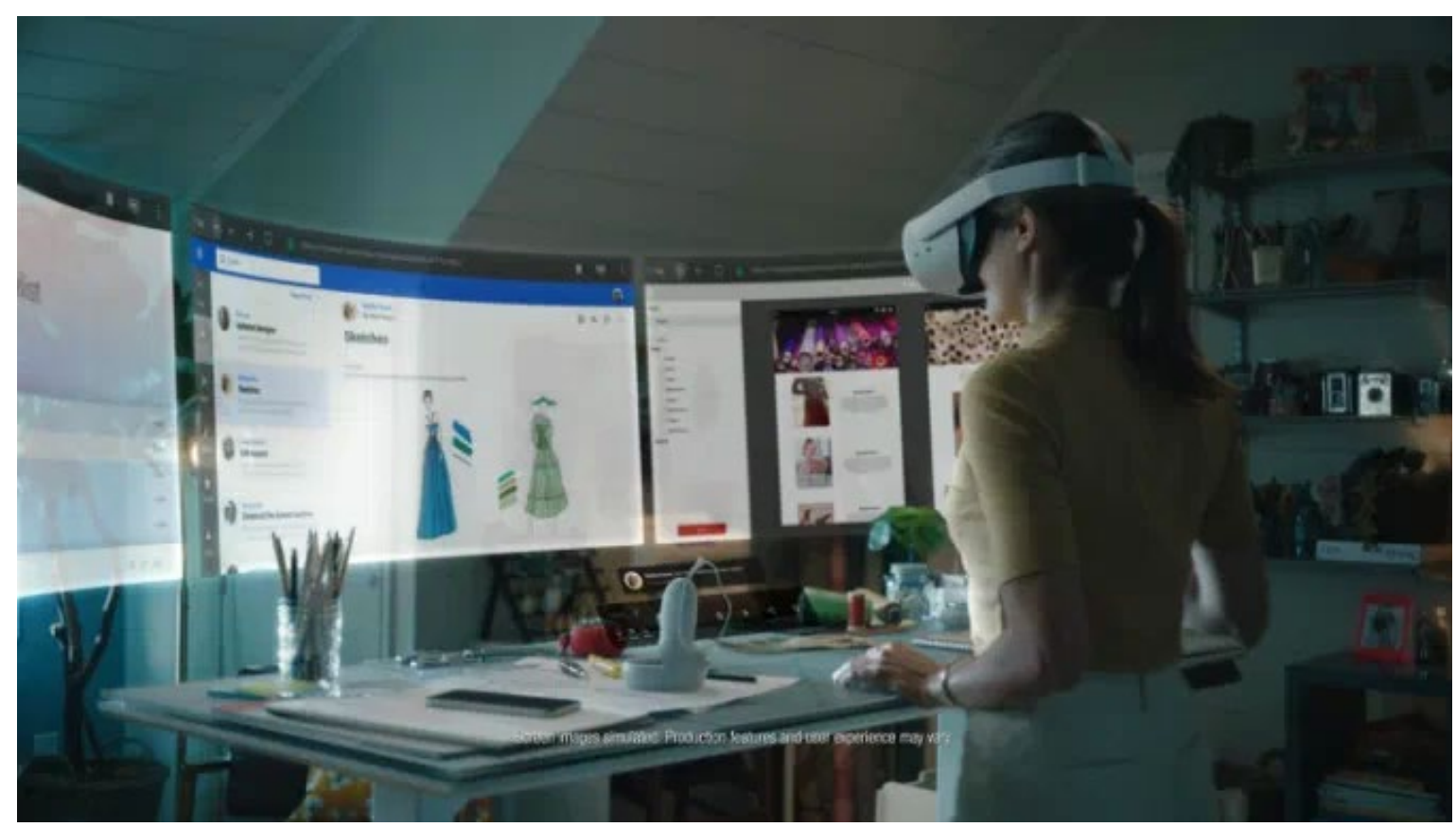

Figure 1 - Screenshot from Facebook's 'Infinite Office' Oculus Quest application, that imagines a mobile workplace fully operationalized within the Facebook platform. 
Eugenic Gaze (via Swauger, 2020) as they may codify xenophobia, ableism and white supremacy behind the black-box of algorithmic bias, "while avoiding equity-based critiques because of our belief in the neutrality of data and technology" (Swauger, 2020).

Viewed through this lens, we see how VR tools are not just a means of immersive simulation, but also of data collection derived from a scenario that is "real" enough to inspire confidence in the data's veracity. Our work consequently warns that VR may become a pervasive site for discrimination. Where VR diverges from other technologies in this regard is in the belief in the capacity of VR to quantify the entire cognitive and physical experience of the user: what we refer to as a fantasy of perfect data.

\section{Productivity Tracking and Analytics in the VR Workplace}

Where we see this issue emerging in the future is in the intersection of VR workplace tools, like Facebook's 'Infinite Office' (see Figure 1) and the data driven 'employee productivity' tracking and analytics apparatus being advanced in workplaces like Amazon warehouses and through software suites like Microsoft 365, which assign individual employees 'productivity scores' based on their use of related software.

What VR - and its fantasy of perfect data - offer this surveillance ensemble is in line with Mark Andrejevic's concept of framelessness, which he uses to describe the contemporary drive of big tech to create a digital 1:1 map of the entire world. This global platform, powered by cameras and sensors everywhere, is intended to serve the purpose of "reflecting the world back to itself in machine readable form" (p. 114) to serve the totalizing ambition of automation.

It is likely that VR will become a site for labour. This may take the form of becoming the replacement for the desktop computer (as 'Infinite Office') imagines, or in other contexts such as customer services delivered over VR chatrooms, or remote telepresence work with robots (discussed below). Our concern is that this offers employers and big tech a new source of data (that is biometric, see Pfeuffer et al. 2019; Miller et al. 2020) for surveillance, algorithmic analysis, and control.

\section{VR, Telepresence and the Gig Economy}

Integrated with this issue of workplace analytics, another potential workplace application for VR that offers significant concern is in the emergence of VR as an interface for teleoperated robots. For instance, in 2020, Japanese grocery chain FamilyMart announced a partnership with robotics company Telexistence involving robots in grocery stores - controlled by remote operators using a VR headset - stocking shelves (Imada, 2020). Separate from the use of autonomous robots, our concern is with the mobilization of VR-enabled robots in the context of precarious labour and the gig economy. FamilyMart's "remote controlled robot 'employees'" were announced with the 'benefit' of allowing "people to have more flexible work schedules", highlighting how the commonly 
discussed 'positives' of VR - such as telepresence and intuitive embodied interfaces may be mobilized to diminish working conditions and create a hyperflexible and mobile workforce, again highlighting the need for greater critical speculation around the new affordances of VR (see also Carter \& Egliston, 2020).

\section{References}

Andrejevic, M. (2019). Automated media. Routledge.

Carter, M. \& Egliston, B. (2020) Ethical Implications of Emerging Mixed Reality Technologies. Socio-Tech Futures Lab.

Carter, M. \& Egliston, B. (2021) What are the risks of Virtual Reality data? Learning Analytics, Algorithmic Bias and a Fantasy of Perfect Data. New Media \& Society.

Corrigan, T. (2018). Making Implicit Methods Explicit: Trade Press Analysis in the Political Economy of Communication. International Journal of Communication, 12, $2751-2772$.

Egliston, B., \& Carter, M. (2020). Oculus imaginaries: The promises and perils of Facebook's virtual reality. New Media \& Society.

Mehrabi, N. et al. (2019). A survey on bias and fairness in machine learning. arXiv preprint arXiv:1908.09635

Miller, M. et al. (2020). Personal identifiability of user tracking data during observation of 360-degree VR video. Scientific Reports 10, 17404.

Pfeuffer, K. et al. (2019). Behavioural biometrics in VR. Conference on Human Factors in Computing Systems - Proceedings, 12.

Swauger, S. (2020). Our Bodies Encoded: Algorithmic Test Proctoring in Higher Education. Hybrid Pedagogy. https://hybridpedagogy.org/our-bodies-encodedalgorithmic-test-proctoring-in-higher-education/

Wasko, J. (2004). The political economy of communications. The SAGE Handbook of Media Studies (pp. 309-330). 